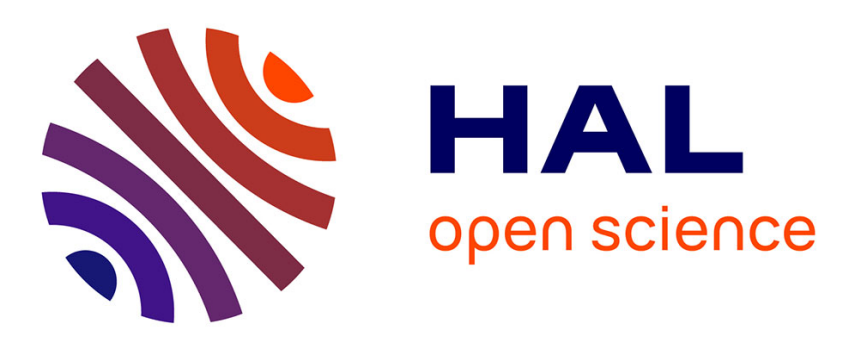

\title{
Control of the bilinear Schrödinger equation for fully coupling potentials
}

Marco Caponigro, Ugo Boscain, Thomas Chambrion, Mario Sigalotti

\section{To cite this version:}

Marco Caponigro, Ugo Boscain, Thomas Chambrion, Mario Sigalotti. Control of the bilinear Schrödinger equation for fully coupling potentials. 18th World Congress of the International Federation of Automatic Control (IFAC), 2011, Milan, Italy. hal-00589363

\section{HAL Id: hal-00589363 https://hal.science/hal-00589363}

Submitted on 28 Apr 2011

HAL is a multi-disciplinary open access archive for the deposit and dissemination of scientific research documents, whether they are published or not. The documents may come from teaching and research institutions in France or abroad, or from public or private research centers.
L'archive ouverte pluridisciplinaire HAL, est destinée au dépôt et à la diffusion de documents scientifiques de niveau recherche, publiés ou non, émanant des établissements d'enseignement et de recherche français ou étrangers, des laboratoires publics ou privés. 


\title{
Control of the bilinear Schrödinger equation for fully coupling potentials
}

\author{
M. Caponigro* U. Boscain ${ }^{* *}$ T. Chambrion ${ }^{*}$ M. Sigalotti* \\ * Institut Élie Cartan, Nancy, France and INRIA Nancy - Grand Est, \\ Projet CORIDA (e-mail: marco.caponigro@inria.fr, \\ thomas.chambrion@iecn.u-nancy.fr, mario.sigalotti@inria.fr) \\ ** CMAP, CNRS, École Polytechnique, Palaiseau, France (e-mail: \\ ugo.boscain@polytechnique.edu).
}

\begin{abstract}
We present a general result of approximate controllability for the bilinear Schrödinger equation (with wave function varying in the unit sphere of an infinite dimensional Hilbert space), under the hypothesis that the Schrödinger operator has discrete spectrum and that the control potential couples all eigenstates. The control method is based on a tracking procedure for the Galerkin approximations, lifted in $S U(n)$. The method allows to estimate the $L^{1}$ norm of the control laws achieving controllability.
\end{abstract}

Keywords: Quantum Control, Control of the Schrödinger equation, Bilinear control systems on Lie groups

\section{INTRODUCTION}

In this paper we are concerned with the controllability problem for the Schrödinger equation

$$
i \frac{d \psi}{d t}=\left(H_{0}+u(t) H_{1}\right) \psi .
$$

Here $\psi$ belongs to the Hilbert sphere of a complex Hilbert space $\mathcal{H}$ and $H_{0}, H_{1}$ are self-adjoint operators on $\mathcal{H}$. The control $u$ is scalar-valued and represents the action of an external field. The most studied case is the one in which $H_{0}=-\Delta+V(x), H_{1}=W(x)$, where $x$ belongs to a domain $D \subset \mathbf{R}^{n}$ with suitable boundary conditions and $V, W$ are functions (identified with the corresponding multiplicative operators) characterizing respectively the autonomous dynamics and the coupling of the system with the control $u$. However, equation (1) can be used to describe more general controlled dynamics. For instance, a quantum particle on a Riemannian manifold subject to an external field (in this case $\Delta$ is the Laplace-Beltrami operator) or a two-level ion trapped in a harmonic potential (the so-called Eberly and Law model Law and Eberly [1996], Bloch et al. [2010]). In the last case, as in many others relevant physical situations, the operator $H_{0}$ cannot be written as the sum of a Laplacian plus a potential.

The controllability problem consists in establishing whether, for every pair of states $\psi_{0}$ and $\psi_{1}$, there exist a control $u(\cdot)$ and a time $T$ such that the solution of (1) with initial condition $\psi(0)=\psi_{0}$ satisfies $\psi(T)=\psi_{1}$. Unfortunately the answer to this problem is negative when $\mathcal{H}$ is infinite dimensional. Indeed, Ball, Marsden, and Slemrod proved in Ball et al. [1982] a result which implies (see Turinici [2000]) that equation (1) is not controllable in (the Hilbert sphere of) $\mathcal{H}$. Moreover, they proved that in the case in which $H_{0}$ is the sum of the Laplacian and a potential in a domain $D$ of $\mathbf{R}^{n}$, equation (1) is neither controllable in the Hilbert sphere $\mathbf{S}$ of $L^{2}(D, \mathbf{C})$ nor in the natural functional space where the problem is formulated, namely the intersection of $\mathbf{S}$ with the Sobolev spaces $H^{2}(D, \mathbf{C})$ and $H_{0}^{1}(D, \mathbf{C})$. Hence one has to look for weaker controllability properties as, for instance, approximate controllability or controllability between the eigenstates of $H_{0}$ (which are the most relevant physical states).

However, in certain cases one can describe quite precisely the set of states that can be connected by admissible paths. Indeed in Beauchard [2005], Beauchard and Coron [2006] the authors prove that, in the case in which $H_{0}$ is the Laplacian on the interval $[-1,1]$, with Dirichlet boundary conditions, and $H_{1}$ is the operator of multiplication by $x$, the system is exactly controllable near the eigenstates in $H^{7}(D, \mathbf{C}) \cap \mathbf{S}$ (with suitable boundary conditions). This result was then refined in Beauchard and Laurent [2010], where the authors proved that the exact controllability holds in $H^{3}(D, \mathbf{C}) \cap \mathbf{S}$, for a large class of control potentials (see also Nersesyan and Nersisyan [2010]).

In dimension larger than one (for $H_{0}$ equal to the sum of the Laplacian and a potential) or for more general situations, the exact description of the reachable set appears to be more difficult and at the moment only approximate controllability results are available.

In Chambrion et al. [2009] an approximate controllability result for (1) was proved via finite dimensional geometric control techniques applied to the Galerkin approximations. The main hypothesis is that the spectrum of $H_{0}$ is discrete and without rational resonances, which means that the gaps between the eigenvalues of $H_{0}$ are Q-linearly independent. Another crucial hypothesis appearing naturally is that the operator $H_{1}$ couples all eigenvectors of $H_{0}$.

The main advantages of that result with respect to those previously known are that: i) it does not need $H_{0}$ to be 
of the form $-\Delta+V$; ii) it can be applied to the case in which $H_{1}$ is an unbounded operator; iii) the control is a bounded function with arbitrarily small bound; iv) it allows to prove controllability for density matrices and it can be generalized to prove approximate controllability results for a system of Schrödinger equations controlled by the same control (see Chambrion [2009]).

Results similar to those presented in Chambrion et al. [2009] have been obtained, with different techniques, in Nersesyan [2010] (see also Ito and Kunisch [2007], Beauchard and Nersesyan [2010], Nersesyan [2009], Nersesyan and Nersisyan [2010]). They require less restrictive hypotheses on the spectrum of $H_{0}$ (which is still assumed to be discrete) but they do not admit $H_{1}$ unbounded and do not work for the density matrices. However, it should be noticed that Nersesyan [2010] proves approximate controllability with respect to some Sobolev norm $H^{s}$, while the results given in Chambrion et al. [2009] permit to get approximate controllability in the weaker norm $L^{2}$. As it happens for the results in Chambrion et al. [2009], the sufficient conditions for controllability obtained in Nersesyan [2010] are generic.

In this paper we prove the approximate controllability of (1) under less restrictive hypotheses on the spectrum of $H_{0}$ than those in Chambrion et al. [2009]. More precisely, assume that $H_{0}$ has discrete spectrum $\left(\lambda_{k}\right)_{k \in \mathbf{N}}$, that the operator $H_{1}$ couples all eigenvectors of $H_{0}$, and that $\mid \lambda_{j}-$ $\lambda_{k}|\neq| \lambda_{l}-\lambda_{m} \mid$ for every $(j, k),(l, m) \in \mathbf{N}^{2} \backslash\{(p, p)$ : $p \in \mathbf{N}\}$ such that $\{j, k\} \neq\{l, m\}$, then the system is approximately controllable.

The idea of the proof is the following. We recover approximate controllability for the system defined on an infinite-dimensional Hilbert space through fine controllability properties of the finite-dimensional Galerkin approximations, which allow us to pass to the limit as $N \rightarrow \infty$. More precisely, we prove that, for $n, N \in \mathbf{N}$ with $N \gg$ $n \gg 1$, for given initial and final conditions $\psi_{0}, \psi_{1}$ in the Hilbert sphere of $\mathcal{H}$ which are linear combinations of the first $n$ eigenvectors of $H_{0}$, it is possible to steer $\psi_{0}$ to $\psi_{1}$ in the Galerkin approximation of order $N$ in such a way that the projection on the last $N-n$ components has arbitrarily small norm along the trajectory. This kind of controllability for the Galerkin approximation of order $N$ is proved in two steps: firstly, thanks to a time-dependent change of variables we transform the system in a driftless one, nonlinear in the control, and we prove the result up to phases. The change of variables was already introduced in Agrachev and Chambrion [2006], Chambrion et al. [2009]; the technical novelty of this paper is the convexification analysis for the transformed system, which allows to conclude the controllability with less restrictive non-resonance hypotheses. Secondly, the control of phases is obtained via a classical method, using as pivot an eigenstate of $H_{0}$ and exploiting the time-reversibility properties of the Schrödinger equation.

\section{FRAMEWORK AND MAIN RESULTS}

\subsection{Settings and notations}

As in Chambrion et al. [2009], we use an abstract framework instead of a presentation in terms of partial dif- ferential equations. The advantage is that this presentation is very versatile and applies without modification for Schrödinger equation on a (possibly not bounded) domain of $\mathbf{R}^{n}$ or on a manifold as $\mathbb{S}^{1}$. Hereafter $\mathbf{N}$ denotes the set of strictly positive integers.

Definition 1. Let $\mathcal{H}$ be an Hilbert space with scalar product $\langle\cdot, \cdot\rangle,(A, B)$ be a pair of (possibly unbounded) linear operators of $\mathcal{H}$, with domains $D(A)$ and $D(B)$, and $\delta>0$. Let us introduce the formal controlled equation

$$
\frac{d \psi}{d t}(t)=(A+u(t) B) \psi(t), \quad u(t) \in[0, \delta] .
$$

We say that $(A, B, \Phi, \delta)$ satisfies ( $\mathfrak{A})$ if the following assumptions are verified:

(A1) $\Phi=\left(\phi_{k}\right)_{k \in \mathbf{N}}$ is an Hilbert basis of $\mathcal{H}$ made of eigenvectors of $A$ associated with the family of eigenvalues $\left(i \lambda_{k}\right)_{k \in \mathbf{N}}$

$(\mathfrak{A} 2) \phi_{k} \in D(B)$ for every $k \in \mathbf{N}$;

(A3) $A+u B: \operatorname{span}\left\{\phi_{k} \mid k \in \mathbf{N}\right\} \rightarrow \mathcal{H}$ is essentially skewadjoint for every $u \in[0, \delta]$;

$(\mathfrak{A} 4)\left\langle\phi_{j}, B \phi_{k}\right\rangle \neq 0$ for every $j \neq k, j, k \in \mathbf{N}$;

$(\mathfrak{A} 5)\left|\lambda_{j}-\lambda_{k}\right| \neq\left|\lambda_{l}-\lambda_{m}\right|$ for every $(j, k),(l, m) \in \mathbf{N}^{2} \backslash$ $\{(p, p): p \in \mathbf{N}\}$ such that $\{j, k\} \neq\{l, m\}$.

A crucial consequence of assumption $(\mathfrak{A} 3)$ is that, for every constant $u$ in $[0, \delta], A+u B$ generates a group of unitary transformations $e^{t(A+u B)}: \mathcal{H} \rightarrow \mathcal{H}$. The unit sphere of $\mathcal{H}$ is invariant for all these transformations.

Definition 2. Let $(A, B, \Phi, \delta)$ satisfy ( $\mathfrak{A})$. For any piecewise constant control function $u:[0, T] \rightarrow[0, \delta]$, the solution of (2) with initial condition $\psi_{0} \in \mathcal{H}$ is

$$
\begin{gathered}
\psi(t)=e^{\left(t-\sum_{l=1}^{j-1} t_{l}\right)\left(A+u_{j} B\right)} \circ e^{t_{j-1}\left(A+u_{j-1} B\right)} \circ \\
\circ \cdots \circ e^{t_{1}\left(A+u_{1} B\right)}\left(\psi_{0}\right),
\end{gathered}
$$

where $\sum_{l=1}^{j-1} t_{l} \leq t<\sum_{l=1}^{j} t_{l}$ and $u(\tau)=u_{j}$ if $\sum_{l=1}^{j-1} t_{l} \leq$ $\tau<\sum_{l=1}^{j} t_{l}$.

Notice that a solution $\psi(\cdot)$ of (2) as in (3) satisfies, for every $n \in \mathbf{N}$ and almost every $t \in[0, T]$ the differential equation

$$
\frac{d}{d t}\left\langle\phi_{n}, \psi(t)\right\rangle=-\left\langle(A+u(t) B) \phi_{n}, \psi(t)\right\rangle .
$$

As already recalled, exact controllability is hopeless in general.

Definition 3. Let $(A, B, \Phi, \delta)$ satisfy $(\mathfrak{A})$. We say that (2) is approximatly controllable if for every $\psi_{0}, \psi_{1}$ in the unit sphere of $\mathcal{H}$ and every $\varepsilon>0$ there exist $k \in \mathbf{N}$, $t_{1}, \ldots, t_{k}>0$, and $u_{1}, \ldots, u_{k} \in U$ such that

$$
\left\|\psi_{1}-e^{t_{k}\left(A+u_{k} B\right)} \circ \cdots \circ e^{t_{1}\left(A+u_{1} B\right)}\left(\psi_{0}\right)\right\|<\varepsilon .
$$

\subsection{Main results}

Theorem 2.1. Let $(A, B, \Phi, \delta)$ satisfy ( $\mathfrak{A})$. Then (2) is approximately controllable.

Theorem 2.2. Let $(A, B, \Phi, \delta)$ satisfy ( $\mathfrak{A})$. For every $\varepsilon>0$ and for every $j, k \in \mathbf{N}, j \neq k$, there exist a piecewise constant control $u:\left[0, T_{u}\right] \rightarrow[0, \delta]$ and $\theta \in \mathbb{S}^{1}$ such that the trajectory $\psi(t)$ of (2) with initial condition $\phi_{j}$ and corresponding to the control $u$ satisfies $\left\|\psi\left(T_{u}\right)-e^{i \theta} \phi_{k}\right\|<\varepsilon$ and

$$
\|u\|_{L^{1}} \leq \frac{5 \pi}{4\left|\left\langle\phi_{j}, B \phi_{k}\right\rangle\right|} .
$$




\section{CONVEXIFICATION PROCEDURE}

\subsection{Time-reparametrization}

We denote by $P C$ the set of piecewise constant functions $u:[0, \infty) \rightarrow[0, \infty)$ such that there exist $u_{1}, \ldots, u_{p}>0$ and $0=t_{1}<\cdots<t_{p+1}=T_{u}$ for which

$$
u: t \mapsto \sum_{j=1}^{p} u_{j} \chi_{\left[t_{j}, t_{j+1}\right)}(t) .
$$

Let us identify $u=\sum_{j=1}^{p} u_{j} \chi_{\left[t_{j}, t_{j+1}\right)}$ with the finite sequence $\left(u_{j}, \tau_{j}\right)_{1 \leq j \leq p}$ where $\tau_{j}=t_{j+1}-t_{j}$ for every $1 \leq j \leq p$.

We define the map

$$
\begin{aligned}
\mathcal{P}: P C & \rightarrow P C \\
\left(u_{j}, \tau_{j}\right)_{1 \leq j \leq p} & \mapsto\left(\frac{1}{u_{j}}, u_{j} \tau_{j}\right),
\end{aligned}
$$

which satisfies the following easily verifiable properties.

Proposition 4. For every $u \in P C, \mathcal{P} \circ \mathcal{P}(u)=u$ and $\|\mathcal{P}(u)\|_{L^{1}}=\sum_{i=1}^{p} \tau_{j}$.

Assume that $(A, B, \Phi)$ satisfies $(\mathfrak{A})$. In analogy with Definition 2, we define, for every $u=\sum_{j=1}^{p} u_{j} \chi_{\left[t_{j}, t_{j+1}\right)} \in P C$ such that $u(t) \in[0, \delta]$ for every $t \geq 0$, the solution of

$$
\frac{d \psi}{d t}(t)=(u(t) A+B) \psi(t)
$$

with initial condition $\psi_{0} \in \mathcal{H}$ as

$$
\psi(t)=e^{\left(t-t_{l}\right)\left(u_{l} A+B\right)} \circ \cdots \circ e^{t_{1}\left(u_{1} A+B\right)}\left(\psi_{0}\right),
$$

where $t_{l} \leq t \leq t_{l+1}$.

System (5) is the time reparametrization of system (2) induced by the transformation $\mathcal{P}$, as stated in the following proposition.

Proposition 5. Let $u=\left(u_{j}, \tau_{j}\right)_{1 \leq j \leq p}$ belong to $P C$ and $\psi_{0}$ be a point of $\mathcal{H}$. Let $\psi$ be the solution of (2) with control $u$ and initial condition $\psi_{0}$, and $\widetilde{\psi}$ be the solution of (5) with control $\mathcal{P}(u)$ and initial condition $\psi_{0}$. Then $\psi\left(T_{u}\right)=\widetilde{\psi}\left(\|u\|_{L^{1}}\right)$.

Proof. It is enough to remark that, if $u \neq 0$, for every $t \in[0, \infty), e^{t(A+u B)}=e^{t u\left(\frac{1}{u} A+B\right)}$.

Remark 6. As a consequence of Proposition 5 it is equivalent to prove controllability for (2) with control $u \in[0, \delta]$ and to prove controllability for system (5) with control $u \in[1 / \delta, \infty)$.

\subsection{Convexification}

For every positive integer $N$ let the matrices $A^{(N)}=$ $\operatorname{diag}\left(i \lambda_{1}, \ldots, i \lambda_{N}\right)$ and

$$
B^{(N)}=\left(\left\langle B \phi_{j}, \phi_{k}\right\rangle\right)_{j, k=1}^{N}=:\left(b_{j k}\right)_{j, k=1}^{N},
$$

be the Galerkyn approximations at order $N$ of $A$ and $B$, respectively. Let $t \mapsto \psi(t)$ be a solution of

$$
\dot{\psi}=\left(u A^{(N)}+B^{(N)}\right) \psi,
$$

corresponding to a control function $u$ and consider $v(t)=$ $\int_{0}^{t} u(\tau) d \tau$. Denote by $\mathrm{d}(B)$ the diagonal of $B^{(N)}$ and let
$\hat{B}^{(N)}=B^{(N)}-\mathrm{d}(B)$. Then $y: t \mapsto e^{-v(t) A^{(N)}-t \mathrm{~d}(B)} \psi(t)$, is a solution of

$$
\dot{y}(t)=e^{-v(t) A^{(N)}-t \mathrm{~d}(B)} \hat{B}^{(N)} e^{v(t) A^{(N)}+t \mathrm{~d}(B)} y(t) .
$$

Let us set $\vartheta_{N}(t, v)=e^{-v A^{(N)}-t \mathrm{~d}(B)} \hat{B}^{(N)} e^{v A^{(N)}+t \mathrm{~d}(B)}$.

Lemma 7. Let $K$ be a positive integer and $\gamma_{1}, \ldots, \gamma_{K} \in$ $\mathbf{R} \backslash\{0\}$ be such that $\left|\gamma_{1}\right| \neq\left|\gamma_{j}\right|$ for $j=2, \ldots, K$. Let

$$
\varphi(t)=\left(e^{i t \gamma_{1}}, \ldots, e^{i t \gamma_{K}}\right) .
$$

Then, for every $t_{0} \in \mathbf{R}$, we have

$$
\overline{\operatorname{conv} \varphi\left(\left[t_{0}, \infty\right)\right)} \supseteq \nu \mathbb{S}^{1} \times\{(0, \ldots, 0)\},
$$

where $\nu=\prod_{k=2}^{\infty} \cos \left(\frac{\pi}{2 k}\right)>0$. Moreover, for every $R>0$ and $\xi \in \mathbb{S}^{1}$ there exists a sequence $\left(t_{k}\right)_{k \in \mathbf{N}}$ such that $t_{k+1}-t_{k}>R$ and

$$
\lim _{h \rightarrow \infty} \frac{1}{h} \sum_{k=1}^{h} \varphi\left(t_{k}\right)=(\nu \xi, 0, \ldots, 0) .
$$

Proof. Since

$$
\varphi\left(t-t_{0}\right)=\left(e^{-i t_{0} \gamma_{1}} e^{i t \gamma_{1}}, \ldots, e^{-i t_{0} \gamma_{K}} e^{i t \gamma_{K}}\right),
$$

it is enough to prove the lemma for $t_{0}=0$. We can suppose that $\left|\gamma_{1}\right|=1$ and, up to a reordering of the indexes, that there exist $n$ and $\tilde{n}$ such that $1 \leq n \leq \tilde{n} \leq K,\left|\gamma_{i}\right| \neq\left|\gamma_{j}\right|$ for every $i, j \in\{1, \ldots, n\}, \gamma_{2}, \ldots, \gamma_{\tilde{n}} \in \mathbf{Z}, \gamma_{\tilde{n}+1}, \ldots, \gamma_{K} \in \mathbf{R} \backslash$ $\mathbf{Z}$, and $\left\{\left|\gamma_{n+1}\right|, \ldots,\left|\gamma_{\tilde{n}}\right|\right\} \subset\left\{\left|\gamma_{2}\right|, \ldots,\left|\gamma_{n}\right|\right\}$.

Consider the $2^{n-1}$ real numbers defined as follows: let

$$
\bar{t}_{1}=0 \text {, }
$$

and for $k \in\{1, \ldots, n-1\}$ and $j \in\left\{1, \ldots, 2^{k-1}\right\}$,

$$
\bar{t}_{2^{k-1}+j}=\bar{t}_{j}+\frac{\pi}{\left|\gamma_{k+1}\right|} \text {. }
$$

Up to a reordering of the $\bar{t}_{j}$, we can suppose that $0=\bar{t}_{1}<$ $\bar{t}_{2}<\cdots<\bar{t}_{2^{n-1}}$. Take an integer $r$ larger than $R / 2 \pi$, then set $t_{j}=\bar{t}_{j}+2 \pi r(j-1)$, in such a way that $t_{k}-t_{k-1}>R$ for every $k=2, \ldots, 2^{n-1}$.

Now consider the arithmetic mean of the $l$-th (complex) coordinates of $\varphi\left(t_{1}\right), \ldots, \varphi\left(t_{2^{n-1}}\right)$. We show that this quantity is zero for $l=2, \ldots, \tilde{n}$. Indeed, from the definition of $t_{j}$, we have

$$
\sum_{j=1}^{2^{n-1}} e^{i t_{j} \gamma_{l}}=\prod_{k=1}^{n-1}\left(1+e^{i \pi \gamma_{l} /\left|\gamma_{k+1}\right|}\right),
$$

which is zero since so is the $k$-th factor when $\left|\gamma_{l}\right|=\left|\gamma_{k+1}\right|$.

On the other hand, the arithmetic mean of the first coordinate is uniformly bounded away from zero. Indeed

$$
\begin{aligned}
\left|\frac{1}{2^{n-1}} \sum_{j=1}^{2^{n-1}} e^{i \gamma_{1} t_{j}}\right| & =\prod_{k=1}^{n-1}\left|\frac{1+e^{i \pi \gamma_{1} /\left|\gamma_{k+1}\right|}}{2}\right| \\
& =\prod_{k=2}^{n} \cos \left(\frac{\pi}{2\left|\gamma_{k}\right|}\right) \\
& =\exp \left(\sum_{k=2}^{n} \log \left(\cos \left(\frac{\pi}{2\left|\gamma_{k}\right|}\right)\right)\right) \\
& \geq \exp \left(\sum_{k=2}^{\infty} \log \left(\cos \left(\frac{\pi}{2 k}\right)\right)\right)=\nu .
\end{aligned}
$$

Since $\log \left(\cos \left(\frac{\pi}{2 k}\right)\right) \sim-\frac{\pi^{2}}{8 k^{2}}$ as $k$ tends to infinity, then the $\operatorname{sum} \sum_{k \geq 2} \log \left(\cos \left(\frac{\pi}{2 k}\right)\right)$ converges to a (negative) finite value $l$. 
Therefore we have found a sequence of numbers $t_{j}$ such that the arithmetic mean of the first coordinate of $\varphi\left(t_{1}\right), \ldots, \varphi\left(t_{2^{n-1}}\right)$ is uniformly bounded away from zero and the arithmetic means of following $\tilde{n}-1$ coordinates are zero. According to $(6)$, the role of $t_{1}, \ldots, t_{2^{n-1}}$ can equivalently be played, for every $k \in \mathbf{N}$, by the $2^{n-1}$-uple

$$
t_{j}^{k}=t_{j}+2 \pi m k, \quad j=1, \ldots, 2^{n-1},
$$

where the integer $m$ is larger than $r+t_{2^{n-1}} / 2 \pi$. Now, let $l \in\{\tilde{n}+1, \ldots, K\}$, so that $\gamma_{l} \notin \mathbf{Z}$. For every $h \in \mathbf{N}$, the arithmetic mean of the $l$-th coordinate of the points $\varphi\left(t_{j}^{k}\right)$ $\left(k=0, \ldots, h, j=1, \ldots, 2^{n-1}\right)$ is

$$
\begin{aligned}
& \frac{1}{2^{n-1}(h+1)} \sum_{j=1}^{2^{n-1}} \sum_{k=0}^{h} e^{i t_{j}^{k} \gamma_{l}}= \\
& =\frac{1}{2^{n-1}(h+1)} \sum_{j=1}^{2^{n-1}} e^{i t_{j} \gamma_{l}} \sum_{k=0}^{h} e^{i 2 \pi m k \gamma_{l}} \\
& =\left(\frac{1}{2^{n-1}} \sum_{j=1}^{2^{n-1}} e^{i t_{j} \gamma_{l}}\right) \frac{1}{(h+1)} \frac{1-e^{i 2 \pi m(h+1) \gamma_{l}}}{1-e^{i 2 \pi m \gamma_{l}}} \stackrel{h \rightarrow \infty}{\longrightarrow} 0 .
\end{aligned}
$$

Therefore, we found a sequence of points in the convex hull of $\varphi([0, \infty))$ converging to $\left(2^{1-n} \sum_{j=1}^{2^{n-1}} e^{i \gamma_{1} t_{j}}, 0, \ldots, 0\right)$. The lemma follows from (7) and by rotation invariance (see $(6))$.

Remark 8 . The constant $\nu$ can be easily computed numerically. One finds $\nu \approx 0.430>\frac{2}{5}$.

\subsection{An auxiliary system}

Let $(A, B, \Phi)$ satisfy $(\mathfrak{A})$. With every $n \in \mathbf{N}$ we associate the control system on $\mathbf{C}^{n}$

$$
\dot{x}=\nu\left|b_{j k}\right|\left(e^{i \theta} e_{j k}^{(n)}-e^{-i \theta} e_{k j}^{(n)}\right) x,
$$

where $\theta=\theta(t) \in \mathbb{S}^{1}$ and $(j, k)=(j(t), k(t)) \in \mathbf{N}^{2}$ $\{(p, p): p \in \mathbf{N}\}$ are piecewise constant controls. Set $e_{j k}^{(n)}$ as the $n \times n$ matrix whose entries are all zero but the one of index $(j, k)$ which is equal to 1 .

The control system $\left(\Sigma_{n}\right)$ is linear in $x$. For every $\theta$ in $\mathbb{S}^{1}$ and every $1 \leq j, k \leq n, j \neq k$, the matrix $e^{i \theta} e_{j k}^{(n)}-e^{-i \theta} e_{k j}^{(n)}$ is skew-adjoint with zero trace. Hence the control system $\left(\Sigma_{n}\right)$ leaves the unit sphere $\mathbf{S}^{n}$ of $\mathbf{C}^{n}$ invariant. In order to take advantage of the rich Lie group structure of group of matrices, it is also possible to lift this system in the group $S U(n)$, considering $x$ as a matrix.

\section{PROOF OF THEOREM 2.1}

\subsection{Controllability of $\left(\Sigma_{n}\right)$}

Proposition 9. Let $(A, B, \Phi, \delta)$ satisfy (A). Then, for every $n$ in $\mathbf{N}$, the finite dimensional control system $\left(\Sigma_{n}\right)$ is completely controllable both in $\mathbf{S}^{n} \subset \mathbf{C}^{n}$ and in $S U(n)$.

Proof. Note that

$$
\left[e_{j k}^{(n)}-e_{k j}^{(n)}, i\left(e_{j k}^{(n)}+e_{k j}^{(n)}\right)\right]=2 i\left(e_{j j}^{(n)}-e_{k k}^{(n)}\right) .
$$

Hence the Lie algebra generated by the linear vector fields $x \mapsto \nu\left|b_{j k}\right|\left(e^{i \theta} e_{j k}-e^{-i \theta} e_{k j}\right) x$ contains the whole tangent space $\mathfrak{s u}(n) x$ of $S U(n)$ at $x$. The controllability in $\mathbf{S}^{n}$ follows by projection.

\subsection{Tracking $\left(\Sigma_{n}\right)$ via $\left(\Theta_{N}\right)$}

Next proposition states that, for every $N \geq n$ system $\left(\Theta_{N}\right)$ can track, in projection on the first $n$ components, without time reparametrization, every trajectory of system $\left(\Sigma_{n}\right)$. Call $\Pi_{n}^{(N)}$ the projection mapping a $N \times N$ complex matrix to the $n \times n$ matrix obtained by dropping the last $N-n$ columns and the last $N-n$ rows.

Proposition 10. For every $n, N \in \mathbf{N}, N \geq n, \varepsilon, \delta>0$, and for every solution $X:[0, T] \rightarrow S U(n)$ of system $\left(\Sigma_{n}\right)$ with initial condition $X(0)=I_{n}$ there exist a piecewise constant control $u:[0, T] \rightarrow[1 / \delta,+\infty)$ such that the solution $Y:[0, T] \rightarrow S U(N)$ of system $\left(\Theta_{N}\right)$ with initial condition $Y(0)=I_{N}$ corresponding to $v(t)=\int_{0}^{t} u(s) d s$ satisfies

$$
\left\|\Pi_{n}^{(N)} Y(t)-X(t)\right\|<\varepsilon, \quad \text { for every } t \in[0, T] .
$$

Proof. Given a trajectory $X(t)$ of system $\left(\Sigma_{n}\right)$ with initial condition $X(0)=I_{n}$, denote by $(j, k)=(j(t), k(t)) \in \mathbf{N}^{2}$, $j \neq k$ and $\theta=\theta(t) \in \mathbb{S}^{1}$ its corresponding control functions. Being these function piecewise constant, it is possible to write $[0, T]$ as union of $q$ intervals $[0, T]=$ $\bigcup_{p=0}^{q}\left[t_{p}, t_{p+1}\right]$ in such a way that $j, k$, and $\theta$ are constant on $\left[t_{p}, t_{p+1}\right)$ for every $p=0, \ldots, q$.

We are going to construct the control $u$ by applying recursively Lemma 7 . Let $\bar{\delta}>1 / \delta$. Fix $p \in\{0, \ldots, q\}$ and $j, k, \theta$ such that $(j(t), k(t))=(j, k)$ and $\theta(t)=$ $\theta$ on $\left[t_{p}, t_{p+1}\right)$. Apply Lemma 7 with $\gamma_{1}=\lambda_{j}-\lambda_{k}$, $\left\{\gamma_{2}, \ldots, \gamma_{K}\right\}=\left\{\lambda_{l}-\lambda_{m} \mid l, m \in\{1, \ldots, N\},\{l, m\} \neq\right.$ $\{j, k\}$, and $l \neq m\}$,

$$
R=\max _{1 \leq l<m \leq n} \frac{\left|b_{l l}-b_{m m}\right|}{\left|\lambda_{l}-\lambda_{m}\right|} T+\bar{\delta} T
$$

and $t_{0}=t_{0}(p)$ to be fixed later depending on $p$. Then, for every $\eta>0$, there exist $h=h(p)>1 / \eta$ and a sequence $\left(w_{\alpha}^{p}\right)_{\alpha=1}^{h}$ such that $w_{1}^{p} \geq t_{0}, w_{\alpha}^{p}-w_{\alpha-1}^{p}>R$, and such that

$$
\left|\frac{1}{h} \sum_{\alpha=1}^{h} e^{i\left(\lambda_{k}-\lambda_{j}\right) w_{\alpha}^{p}}-\nu \frac{\bar{b}_{j k}}{\left|b_{j k}\right|} e^{i \theta}\right|<\eta,
$$

and

$$
\left|\frac{1}{h} \sum_{\alpha=1}^{h} e^{i\left(\lambda_{l}-\lambda_{m}\right) w_{\alpha}^{p}}\right|<\eta
$$

for every $l, m \in\{1, \ldots, N\}$ such that $\{l, m\} \neq\{j, k\}$, and $l \neq m$.

Set $\tau_{\alpha}^{p}=t_{p}+\left(t_{p+1}-t_{p}\right) \alpha / h, \alpha=0, \ldots, h$, and define the piecewise constant function

$$
v_{\eta}(t)=\sum_{p=0}^{q} \sum_{\alpha=1}^{h}\left(w_{\alpha}^{p}+i \tau_{\alpha}^{p} \frac{b_{j j}-b_{k k}}{\lambda_{j}-\lambda_{k}}\right) \chi_{\left[\tau_{\alpha-1}^{p}, \tau_{\alpha}^{p}\right)}(t) .
$$

Note that by choosing $t_{0}(p)=w_{h(p-1)}^{p-1}+R$ for $p=1, \ldots, q$ and $t_{0}(0)=R$ we have that $v_{\eta}(t)$ is non-decreasing.

Set $M(t)=\nu\left|b_{j(t) k(t)}\right|\left(e^{i \theta(t)} e_{j(t) k(t)}^{(N)}-e^{-i \theta(t)} e_{k(t) j(t)}^{(N)}\right)$. The definition of $v_{\eta}$ is such that

$$
\int_{0}^{t} \vartheta_{N}\left(s, v_{\eta}(s)\right) d s \stackrel{\eta \rightarrow 0}{\longrightarrow} \int_{0}^{t} M(s) d s,
$$

uniformly with respect to $t \in[0, T]$. This convergence guarantees (see for example [Agrachev and Sachkov, 2004, 
Lemma 8.2]) that the projection $\Pi_{n}^{(N)}$ of the solution $Y_{\eta}(t)$ of system $\left(\Theta_{N}\right)$ with control $v_{\eta}$ and initial condition $I_{N}$ converges uniformly in $[0, T]$ to $X(t)$ as $\eta$ tends to 0 . In particular it is possible to choose $\eta$ sufficiently small in such a way that

$$
\left\|\Pi_{n}^{(N)} Y_{\eta}(t)-X(t)\right\|<\varepsilon / 2 \quad \text { for every } t \in[0, T] .
$$

If $v_{\eta}(t)$ were of the type $\int_{0}^{t} u(s) d s$ for $u:[0, T] \rightarrow[1 / \delta, \infty)$ piecewise constant then we would be done. Although $v_{\eta}$ is piecewise constant, it is possible to construct a continuous piecewise linear approximation $v_{\eta}^{\prime}$ of $v_{\eta}$ with slope not smaller than $\bar{\delta}$ and such that

$$
\int_{0}^{t}\left(\vartheta_{N}\left(\tau, v_{\eta}(\tau)\right)-\vartheta_{N}\left(\tau, v_{\eta}^{\prime}(\tau)\right)\right) d \tau \stackrel{\eta \rightarrow 0}{\longrightarrow} 0,
$$

uniformly with respect to $t \in[0, T]$. This is possible thanks to the choice of $R$ and since the size of every interval $\left[\tau_{k}^{p}, \tau_{k+1}^{p}\right]$ is smaller that $1 / h<\eta$.

In particular there exists $\eta$ sufficiently small in such a way that, if $Y_{\eta}^{\prime}$ is the solution of system $\left(\Theta_{N}\right)$ with control $v_{\eta}^{\prime}$ and initial condition $I_{N}$, then

$$
\left\|Y_{\eta}(t)-Y_{\eta}^{\prime}(t)\right\|<\varepsilon / 2 \quad \text { for every } t \in[0, T] .
$$

Finally, construct the control $u$ as as the derivative of $v_{\eta}^{\prime}$.

\subsection{Tracking $\left(\Sigma_{n}\right)$ in modulus via the original system}

Next proposition extend the result of tracking for the system $\left(\Theta_{N}\right)$ to the infinite dimensional system (5).

Proposition 11. For every $\varepsilon>0$, for every integer $n$, and for every trajectory $x:[0, T] \rightarrow \mathbf{S}^{n}$ of system $\left(\Sigma_{n}\right)$ there exists a piecewise constant control function $u$ : $[0, T] \rightarrow[1 / \delta,+\infty)$ and a corresponding trajectory $\psi(t)$ of (5) satisfying ||$\left\langle\phi_{j}, \psi(t)\right\rangle|-| x_{j}(t)||<\varepsilon$ for every $t$ in $[0, T], j=1, \ldots, n$ and $\left|\left\langle\phi_{j}, \psi(0)\right\rangle-x_{j}(0)\right|<\varepsilon$ for every $j=1, \ldots, n$.

Proof. Consider $\mu>0$. For every $j \in \mathbf{N}$ the hypothesis that $\phi_{j}$ belongs to $D(B)$ implies that the sequence $\left(b_{j k}\right)_{k \in \mathbf{N}}$ is in $\ell^{2}$. It is therefore possible to choose $N \geq n$ such that $\sum_{k>N}\left|b_{j k}\right|^{2}<\mu$ for every $j=1, \ldots, n$.

Let $X:[0, T] \rightarrow S U(n)$ be the solution of system $\left(\Sigma_{n}\right)$ corresponding to the control associated with the trajectory $x$ and with initial condition $X(0)=I_{n}$. By Proposition 10, for every $\eta>0$ there exist a piecewise constant control $u^{\eta}:[0, T] \rightarrow[1 / \delta,+\infty)$ such that the solution $Y:$ $[0, T] \rightarrow S U(N)$ of system $\left(\Theta_{N}\right)$ with initial condition $I_{N}$ corresponding to $v^{\eta}(t)=\int_{0}^{t} u^{\eta}(\tau) d \tau$ satisfies

$$
\left\|\Pi_{n}^{(N)} Y(t)-X(t)\right\|<\eta,
$$

for every $t \in[0, T]$. Notice that $Y(t)=R^{\eta}(t, 0)$ is the resolvent of $\left(\Theta_{N}\right)$ associated with the control $v^{\eta}$.

Let $\psi(t)$ be the solution of (5) corresponding to $u^{\eta}$ with initial condition $\psi(0)=x_{1}(0) \phi_{1}+\cdots+x_{n}(0) \phi_{n}$. Call

$$
q_{j}^{\eta}(t)=e^{-i \lambda_{j} v^{\eta}(t)-t b_{j j}}\left\langle\phi_{j}, \psi(t)\right\rangle, \quad j \in \mathbf{N} .
$$

For almost every $t \in[0, T]$ and for every $j \in \mathbf{N}$,

$$
\dot{q}_{j}^{\eta}(t)=\sum_{k=1}^{\infty} b_{j k} e^{i\left(\lambda_{k}-\lambda_{j}\right) v^{\eta}(t)+t\left(b_{k k}-b_{j j}\right)} q_{k}^{\eta}(t) .
$$

Therefore $Q_{N}^{\eta}(t)=\left(q_{1}^{\eta}(t), \ldots, q_{N}^{\eta}(t)\right)^{T}$ satisfies the timedependent linear equation

$$
\dot{Q}_{N}^{\eta}(t)=\vartheta_{N}\left(t, v^{\eta}(t)\right) Q_{N}^{\eta}(t)+P_{N}^{\eta}(t),
$$

with $\left\|\Pi_{n}^{(N)} P_{N}^{\eta}(t)\right\| \leq \sqrt{\mu n}$. Hence

$$
Q_{N}^{\eta}(t)=R^{\eta}(t, 0)\left(\begin{array}{c}
x(0) \\
0
\end{array}\right)+\int_{0}^{t} R^{\eta}(s, t) P_{N}^{\eta}(s) d s .
$$

Consider the projection on the first $n$ coordinates. By (9), $R^{\eta}(s, t)$ converges uniformly, as $\eta$ tends to 0 , to a time dependent operator from $\mathbf{C}^{N}$ into itself which preserves the norm of the first $n$ components, then there exists $\eta$ sufficiently small such that

$$
\left\|\Pi_{n}^{(N)}\left(\int_{0}^{t} R^{\eta}(t, s) P_{N}^{\eta}(s) d s\right)\right\|<2 T \sqrt{\mu n} .
$$

Hence

$$
\left\|Q_{n}^{\eta}(t)-x(t)\right\|<\varepsilon,
$$

for $\eta$ and $\mu$ small enough. In particular, for $j=1, \ldots, n$

$$
||\left\langle\phi_{j}, \psi(t)\right\rangle|-| x_{j}(t)||=|| q_{j}^{\eta}(t)|-| x_{j}(t)||<\varepsilon .
$$

\subsection{Approximate controllability}

For $n \in \mathbf{N}$, let $\pi_{n}: \mathcal{H} \rightarrow \mathbf{C}^{n}$ be the map associating with $\psi \in \mathcal{H}$ the $n$-uple $\left(\left\langle\phi_{1}, \psi\right\rangle, \ldots,\left\langle\phi_{n}, \psi\right\rangle\right)$.

Approximate controllability follows from Proposition 11 and Remark 6 applied both to (2) and to its time-reversed version.

Proof of Theorem 2.1. Fix $\psi_{0}, \psi_{1} \in \mathbf{S}, \varepsilon>0$, and $n$ sufficiently large such that $\psi_{0}$ is $\varepsilon$-close to $\sum_{j=1}^{n}\left\langle\phi_{j}, \psi_{0}\right\rangle \phi_{j}$. Without loss of generality we can assume that $\lambda_{1} \neq 0$.

Thanks to Proposition 9, there exists an admissible trajectory $x$ of $\left(\Sigma_{n}\right)$ steering $\pi_{n}\left(\psi_{0}\right) \varepsilon$-close to $\pi_{n}\left(\phi_{1}\right)=$ $(1,0, \ldots, 0)$. Applying Proposition 11 and Remark 6 , there exists a piecewise constant control function $u:[0, T] \rightarrow$ $[0, \delta]$ and a corresponding trajectory $\psi(t)$ of (2) satisfying ||$\left\langle\phi_{j}, \psi(t)\right\rangle|-| x_{j}(t)||<\varepsilon$ for every $t$ in $[0, T], j=1, \ldots, n$ and $\left|\left\langle\phi_{j}, \psi(0)\right\rangle-x_{j}(0)\right|<\varepsilon$ for every $j=1, \ldots, n$.

In particular, taking $t=0, T$, we have that both $\| \psi(0)-$ $\psi_{0} \|<2 \varepsilon$ and $\left\|\psi(T)-e^{i \theta} \phi_{1}\right\|<C \varepsilon$, for some $\theta \in \mathbb{S}^{1}$, with $C$ independent of $n, \varepsilon$ and $\psi_{0}$.

Hence, thanks to the unitarity of the evolution of (2), $u$ steers $\psi_{0}$ to a $(C+2) \varepsilon$-neighborhood of $e^{i \theta} \phi_{1}$.

Note that if $(A, B, \Phi, \delta)$ satisfies $(\mathfrak{A})$, then the same is true for $(-A,-B, \Phi, \delta)$. Notice, moreover, that, by unitarity of the evolution of $(2)$, if $u:[0, T] \rightarrow[0, \delta]$ steers $\psi_{0}$ to a $\varepsilon$-neighborhood of $\psi_{1}$ for the time-reversed control system $\dot{\psi}(t)=-(A+u(t) B) \psi(t)$, then $u(T-\cdot):[0, T] \rightarrow[0, \delta]$ steers $\psi_{1} \varepsilon$-close to $\psi_{0}$ for the original system (2).

Hence there exists a control function $\tilde{u}:[0, \tilde{T}] \rightarrow[0, \delta]$ steering (2) from $e^{i \tilde{\theta}} \phi_{1}$ to a $(C+2) \varepsilon$-neighborhood of $\psi_{1}$, for some $\tilde{\theta} \in \mathbb{S}^{1}$.

Let $\tau>0$ be such that

$$
e^{\tau A}\left(e^{i \theta} \phi_{1}\right)=e^{i \tilde{\theta}} \phi_{1} .
$$

Finally, the concatenation of $u$, of the control constantly equal to zero for a time $\tau$, and of $\tilde{u}(\tilde{T}-\cdot)$ steers (2) from $\psi_{0}$ to a $2(C+2) \varepsilon$-neighborhood of $\psi_{1}$. 


\section{ESTIMATES OF THE $L^{1}$ NORM}

We derive now estimates of the minimal $L^{1}$ norm of the control $u$ steering $\phi_{j}$ to a $\varepsilon$-neighborhood of $\phi_{k}$.

The strategy to get $L^{1}$ estimates is the following. Recall that, instead of considering the control system $\dot{x}=(A+$ $u B) x$ driven by a piecewise constant function $u:\left[0, T_{u}\right] \rightarrow$ $[0, \delta]$, we have defined the function $\mathcal{P}(u):\left[0,\|u\|_{L^{1}}\right] \rightarrow$ $[1 / \delta, \infty)$ and considered the control system $\dot{x}=(\mathcal{P}(u) A+$ $B) x$. To estimate the $L^{1}$ norm of $u$, it is enough to estimates the time needed to transfer the system $\dot{x}=$ $(u A+B) x$ from a given source to a given target. We observe that, in view of Proposition 11, the time needed for the transfer of $\dot{x}=(u A+B) x$ from $\phi_{j}$ to an $\varepsilon$ neighborhood of $\phi_{k}$ is smaller than or equal to the time needed to transfer system $\left(\Sigma_{n}\right)$ from $\pi_{n}\left(\phi_{j}\right)$ to an $\varepsilon$ neighborhood of $\pi_{n}\left(\phi_{k}\right)(n \geq j, k)$.

Such time estimates have been given for general trajectories (i.e., trajectories that do not necessarily steer an eigenstate of $A$ to another) in Agrachev and Chambrion [2006].

We proceed now to the proof of the Theorem 2.2.

Proof of Theorem 2.2. The solution $x: \mathbf{R} \rightarrow \mathbf{S}^{n}$ of the differential equation

$$
\dot{x}=\nu\left|b_{j k}\right|\left(e_{j k}^{(n)}-e_{k j}^{(n)}\right) x
$$

with initial condition $x(0)=\pi_{n}\left(\phi_{j}\right)$ is a trajectory of $\left(\Sigma_{n}\right)$. For every $t$ in $\mathbf{R}, x(t)=\exp \left(t \nu\left|b_{j k}\right|\left(e_{j k}^{(n)}-e_{k j}^{(n)}\right)\right) \pi_{n}\left(\phi_{j}\right)$. In particular, one checks that $x\left(\frac{\pi}{2 \nu\left|b_{j k}\right|}\right)=-\pi_{n}\left(\phi_{k}\right)$.

In other words, the control system $\left(\Sigma_{n}\right)$ can exchange (up to a phase factor) the eigenstates $j$ and $k$ of $A$, in time less than $\frac{\pi}{2 \nu\left|b_{j k}\right|}<\frac{5 \pi}{4\left|b_{j k}\right|}$.

Remark 12. Notice that the bound given in Theorem 2.2 does not depend on $\varepsilon$. However, it is possible that the time $T_{u}$ needed to achieve the transfer of system (2) grows to infinity as $\varepsilon$ tends to zero. When only some $\left|b_{j k}\right|$ are small, the bound given by Theorem 2.2 may be too conservative. It is possible to find other estimates by comparing the time needed to steer an eigenstate to another with ad-hoc metrics on $S U(n)$. The resulting expressions are somewhat intricate and can be found in [Agrachev and Chambrion, 2006, Section 5].

\section{ACKNOWLEDGEMENTS}

This work was supported by the ANR project GCM, program "Blanche", project number NT09_504490 and by the European Research Council, ERC StG 2009 "GeCoMethods", contract number 239748. The article has been partially written while attending the trimester "Control of Partial Differential Equations and Applications" held at IHP in Paris in 2010.

\section{REFERENCES}

Andrei Agrachev and Thomas Chambrion. An estimation of the controllability time for single-input systems on compact Lie groups. ESAIM Control Optim. Calc. Var., 12(3):409-441, 2006. ISSN 1292-8119.
Andrei A. Agrachev and Yuri L. Sachkov. Control theory from the geometric viewpoint, volume 87 of Encyclopaedia of Mathematical Sciences. Springer-Verlag, Berlin, 2004. ISBN 3-540-21019-9. Control Theory and Optimization, II.

J. M. Ball, J. E. Marsden, and M. Slemrod. Controllability for distributed bilinear systems. SIAM J. Control Optim., 20(4):575-597, 1982. ISSN 0363-0129.

Karine Beauchard. Local controllability of a 1-D Schrödinger equation. J. Math. Pures Appl. (9), 84(7): 851-956, 2005. ISSN 0021-7824.

Karine Beauchard and Jean-Michel Coron. Controllability of a quantum particle in a moving potential well. $J$. Funct. Anal., 232(2):328-389, 2006. ISSN 0022-1236.

Karine Beauchard and Camille Laurent. Local controllability of 1D linear and nonlinear Schrödinger equations with bilinear control. J. Math. Pures et Appl., 94(5): 520-554, 2010.

Karine Beauchard and Vahagn Nersesyan. Semi-global weak stabilization of bilinear Schrödinger equations. arXiv:1005.4558v1, 2010.

Anthony M. Bloch, Roger W. Brockett, and Chitra Rangan. Finite controllability of infinite-dimensional quantum systems. IEEE Trans. Automat. Control, 55(8): 1797-1805, 2010.

Thomas Chambrion. Simultaneous approximate tracking of density matrices for a system of Schroedinger equations. arXiv:0902.3798v1, 2009.

Thomas Chambrion, Paolo Mason, Mario Sigalotti, and Ugo Boscain. Controllability of the discrete-spectrum Schrödinger equation driven by an external field. Ann. Inst. H. Poincaré Anal. Non Linéaire, 26(1):329-349, 2009. ISSN 0294-1449.

Kazufumi Ito and Karl Kunisch. Optimal bilinear control of an abstract Schrödinger equation. SIAM J. Control Optim., 46(1):274-287, 2007. ISSN 0363-0129.

C. K. Law and J. H. Eberly. Arbitrary control of a quantum electro-magnetic field. Phys. Rev. Lett., 76(7): 1055-1058, 1996.

Vahagn Nersesyan. Growth of Sobolev norms and controllability of the Schrödinger equation. Comm. Math. Phys., 290(1):371-387, 2009. ISSN 0010-3616.

Vahagn Nersesyan. Global approximate controllability for Schrödinger equation in higher Sobolev norms and applications. Ann. Inst. H. Poincaré Anal. Non Linéaire, 27(3):901-915, 2010. ISSN 0294-1449.

Vahagn Nersesyan and Hayk Nersisyan. Global exact controllability in infinite time of Schrödinger equation. arXiv:1006.2602v1, 2010.

Gabriel Turinici. On the controllability of bilinear quantum systems. In M. Defranceschi and C. Le Bris, editors, Mathematical models and methods for ab initio Quantum Chemistry, volume 74 of Lecture Notes in Chemistry. Springer, 2000. 